\title{
Cochlear Implant Children Auditory Skills: A Study
}

\author{
R. Sundaresan ${ }^{1}$ DQGIA. Turin Martina ${ }^{2}$ \\ ${ }^{1}$ Research Scholar, ${ }^{2}$ Research Supervisor and Associate Professor, PG and Research Department of Rehabilitation Science \\ Holy Cross College (Autonomous), Tiruchirappalli, Tamil Nadu, India \\ E-Mail: sundaresansl@yahoo.com
}

\begin{abstract}
Cochlear implants are now established as the treatment of choice in the rehabilitation of children with bilateral profound sensori neural hearing impairment. This study objective is to analyze and the development of auditory skills for the cochlear implantation children. This study included 50 children attending the Therapy centre, Tiruchirappalli. Their age ranged below 24 months. All children had bilateral sensori-neural hearing loss since birth. None of the subjects had prior Cochlear implant, but all had tried conventional hearing aid. All subjects were implanted unilateral and bilateral, sequential/simultaneous with CI devices including MED EL and Nucleus. All met the criteria of cochlear implant. All items of the studied auditory skills improved significantly by time. There is significant improvement between 4 to 8 months in the scores of detection, discrimination, identification and comprehension skills. Significant improvement in comprehension is also found between 10 and 11 months interval. PAS score had weak negative correlation with age. Children fitted with cochlear implants showed significant improvement in acquisition of auditory skills over a period of below 1year. The age and the side of the implant are the most significant factors.

Keywords: Cochlear Implant, Children Auditory Skills, Language Skills
\end{abstract}

\section{INTRODUCTION}

Birth of a deaf child in a family arise new and challenging problems for parents. Parents of children with disabilities may experience chronic stress. The deaf children are unable to speak and communicate with other people, due to their hearing impairment. Medical care and educational needs can impact the adaptability of families with deaf children and have some important consequences such as social, economic, and emotional restrictions in the family (Arnaud et al., 2008). Cochlear implants are now firmly established as the first treatment of choice in the rehabilitation of individuals with bilateral, profound sensori neural hearing impairment. There is growing evidence that early application of cochlear implant is of great importance for the development of adequate auditory performance and language skills.

Auditory thresholds of the cochlear implanted children allow access to the auditory information beyond that available to the deaf child who routinely use the conventional amplification that offer a critical substrate for auditory therapy. Blamey et al. reported that age of implantation, amount of residual hearing, socio economic status, and mode of communication, nonverbal intelligence and family support are potential predictors of the outcome.
To evaluate the auditory skill development of very young children is challenging due to their limited response capabilities with traditional assessments of development. Some studies have elected to use questionnaires or rating scales of auditory development, such as the Category of Auditory Performance (CAP) or the Infant-Toddler Meaningful Auditory Integration Scale (IT-MAIS) and the Auditory Skills Checklist Other studies have used head turn procedures and/or visual looking preferences to assess auditory discrimination abilities.

The aim of the present study is to track the development of auditory skills in children fitted with cochlear implant, in order to reach a better understanding of the pattern of their development and to study some of the variables associated with difference in the outcome following cochlear implant.

\section{METHODS AND MATERIALS}

Fifty children were included in this study. Their age ranged between 12 months old. The children were attendant to the Therapy centre, Tiruchirappalli, Tamil Nadu. All children had pre-lingual onset of bilateral sensor neural hearing loss. None of the subjects had prior Cochlear implant, but all had tried conventional hearing aid. Range of duration of hearing loss was since birth and duration of implants is 12 months on average. All subjects were implanted either unilaterally or bilaterally, sequential and simultaneous with CI devices including MED EL and Nucleus. All met the criteria of cochlear implant. None of the children has any retro cochlear pathology or cochlear anomalies. All children have average IQ and good physical fitness. They were compared using t-test. Two tailed significant values were considered when $\mathrm{p}<0.05$. Data were analyzed using SPSS for windows version 21.

\section{DATA ANALYSIS AND INTERPRETATION}

Table I Personal Profile Of The Respondents

\begin{tabular}{|l|c|c|}
\hline \multicolumn{1}{|c|}{ Particulars } & No. of Respondents & Percentage \\
\hline \multicolumn{3}{|c|}{ Age } \\
\hline 1 to 12 months & 29 & 58 \\
\hline 13 to 24 months & 21 & 42 \\
\hline \multicolumn{3}{|c|}{ Sex } \\
\hline Male & 22 & 44 \\
\hline Female & 28 & 56 \\
\hline
\end{tabular}


TABLE II PAS SCORE

\begin{tabular}{|l|c|c|}
\hline & $\begin{array}{c}\text { Correlation } \\
\text { Value }\end{array}$ & Statistical Inference \\
\hline Age & .891 & $.079>0.05$ Not Significant \\
\hline Sex & .927 & $.121>0.05$ Not Significant \\
\hline Side of implant & .647 & $.001<0.05$ Significant \\
\hline
\end{tabular}

Cochlear implants provide an improved auditory signal and enhance the development of speech-perception and production skills for profoundly deaf children. However exactly when these early speech skills begin to develop remains unclear. The present study aimed at evaluating. Development of auditory skills in children fitted with cochlear implant over a period of 12 month.

The results revealed significant improvement in all items of the studied auditory skills. There was significant improvement between 3-6 months interval in the scores of the following skills detection, discrimination, identification and comprehension. Significant improvement in comprehension is also found between 6 and 9 months; and between 9 and 12 months interval.

In this study we assessed the possible effects of age, gender and side of implants variables are development of the auditory skills. We found that there was a significant correlation of the outcome with the age and the side of the implant. On the other hand, in-significant correlation with the type of implant and the gender of the child. Regarding the age, our data revealed that children who were implanted at a younger age were more likely to achieve better results. Possible explanation might be related to neural plasticity, early implantation, increase the access to sound during this sensitive period of development (the first 2 years of life).

\section{CONCLUSION AND RECOMMENDATIONS}

Present data revealed significant correlation of the outcome with the age of the child, side of implant and insignificant correlation with the type of implant and children's sex. Children fitted with cochlear implants showed significant improvement in acquisition of auditory skills over a period of 12 months. An assessment protocol for auditory skills program should be formulated in Tamil language. Determining the long-term impact in implanted children requires additional study with larger groups of subjects, different age groups and more prolonged monitoring.
Progress of the therapy (for early intervention providers) needs to be monitored to determine whether the intervention are yielding the anticipated progress and to evaluate areas of strength and weakness of children during aural rehabilitation. Parent written report is recommended to describe auditory skills progress. Rehabilitation course should be increased and be more intensive for those who show slow progress.

\section{REFERENCES}

[1] Archbold S., et al. (1995). Categories of Auditory Performance. Ann. Otol. Rhinol. Laryngol. Suppl., 166, 312-4.

[2] Arnaud, C., et al. (2008). Parent-reported quality of life of children with cerebral palsy in Europe. Pediatrics, 121(1), 54-64.

[3] Beer, J.et al. (2012), Auditory skills, language development, and adaptive behavior of children with cochlear implants and additional disabilities. Int.J. Audiol., 51(6), 491-8.

[4] Blamey P. J. et al. (2001), Relationships among speech perception, production, language, hearing loss, and age in children with impaired hearing. J. Speech Lang. Hear. Res., 44(2), 264-85.

[5] Esser-Leyding B. \& Anderson I. (2012), Ears (Evaluation of Auditory Responses to Speech): An internationally validated assessment tool for children provided with cochlear implants. ORL. $J$. Otorhinolaryngol. Relat. Spec., 74(1), 42-51.

[6] Gabrilo T.E., et al. (1996), Early speech changes in children with multichannel cochlear implants. Otolaryngol. Head Neck Surg., 115(6), 508-12.

[7] Geers A. E. (2004), Speech, language, and reading skills after early cochlear implantation. Arch. Otolaryngol. Head Neck. Surg., 130(5), 634-8.

[8] Mcconkey Robbins A. et al. (2004), Effect of age at cochlear implantation on auditory skill development in infants and toddlers. Arch. Otolaryngol. Head Neck Surg., 130(5), 570-4.

[9] Meinzen-derr J. et al. (2007), Auditory Skills Checklist: Clinical tool for monitoring functional auditory skill development in young children with cochlear implants. Ann. Otol. Rhinol. Laryngol., 116(11), 812-8.

[10] Miyamoto R. T. et al. (2008), Language skills of profoundly deaf children who received cochlear implants under 12 months of age: A preliminary study. Acta. Otolaryngol., 128(4), 373-7.

[11] Miyamoto R. T., Houston D. M. \& Bergeson T. (2005), Cochlear implantation in deaf infants. Laryngoscope, 115(8), 1376-80.

[12] Peters B. R., Litovsky R., Parkinson A., et al. (2007), Importance of age and post implantation experience on speech perception measures in children with sequential bilateral cochlear implants. Otol. Neurotol., 28(5), 649- 57.

[13] Peterson N. R., Pisoni D. B. \& Miyamoto R. T. (2010), Cochlear implants and spoken language processing abilities: review and assessment of the literature. Restor Neurol. Neurosci., 28(2), 237-50.

[14] Tomblin J.B., et al. (2005), The effect of age at cochlear implant initial stimulation on expressive language growth in infants and toddlers. J. Speech Lan. Hear Res., 48(4), 853-67.

[15] Waltzman S. B. and Roland J. T. J. R. (2005, October), Cochlear implantation in children younger than 12 months. Pediatrics. 116(4), 487-93. 